\title{
Absence of Malat1 does not prevent DEN-induced hepatocarcinoma in mice
}

\author{
STÉPHANIE MIARD $^{1}$, MARIE-JOSÉE GIRARD $^{1,2}$, PHILIPPE JOUBERT ${ }^{1}$, SOPHIE CARTER $^{1,2}$, AMY GONZALES $^{3}$, \\ HUIPING GUO $^{3}$, BENJAMIN MORPURGO $^{3}$, LOUISE BOIVIN ${ }^{1}$, ANDREI GOLOVKO $^{3}$ and FRÉDÉRIC PICARD ${ }^{1,2}$ \\ ${ }^{1}$ Research Center, Quebec Heart and Lung Institute; ${ }^{2}$ Faculty of Pharmacy, Laval University, Québec, \\ QC G1V 4G5, Canada; ${ }^{3}$ Texas A\&M Institute for Genomic Medicine, College Station, TX 77843, USA
}

Received August 25, 2016; Accepted January 12, 2017

DOI: $10.3892 /$ or.2017.5468

\begin{abstract}
Long non-coding RNAs (lncRNA) are key regulators of gene expression both at the transcriptional and post-transcriptional levels. The lncRNA metastasis associated lung adenocarcinoma transcript 1 (Malat1) is overexpressed in many types of cancer, including hepatocarcinoma, and induces cell proliferation in several cell lines in vitro. However, the direct causal effects of Malat1 on hepatocyte proliferation and liver carcinogenesis in vivo are not fully understood. To better determine the contribution of Malat1 to hepatocarcinoma oncogenesis, this study was aimed at testing the hypothesis that its absence confers resistance to the development of liver tumors. Male Malat $1^{-/}$mice and their wild-type (WT) littermates were studied one year after treatment with the genotoxic agent diethylnitrosamine (DEN), a potent inducer of liver cancer. As expected, in WT mice, Malat1 expression was significantly higher in hepatic tumors than in healthy liver regions. Altered hepatic mRNA levels of Ki67, HDAC3, NFkB and p27 were observed in DEN-treated Malat ${ }^{-/-}$mice. Despite this, these mice were characterized by similar liver weight, prevalence of tumors, and histological features compared to those of their WT littermates. In parallel, plasma lipids and glucose homeostasis did not significantly differ between DEN-treated groups. These findings support a role for Malat1 as a marker of liver carcinogenesis, but also suggest that its role in the regulation of hepatocyte hyperproliferation in mice is either minimal or masked by redundant and/or overwhelming mechanisms.
\end{abstract}

\section{Introduction}

Human and mouse genomes generate many transcripts that lack the capacity to produce functional proteins. These products include non-coding RNAs (ncRNA) (1). Small

Correspondence to: Dr Frédéric Picard, Research Center, Quebec Heart and Lung Institute, Y4255.3 Pavillon Marguerite-d'Youville, 2725 Chemin Ste-Foy, Québec, QC G1V 4G5, Canada

E-mail: frederic.picard@criucpq.ulaval.ca

Key words: long non-coding RNA, Malat1, cancer, proliferation, DEN, genotoxicity
( $<200$ nucleotides) ncRNAs are involved in multiple biological processes such as neuronal development, and are potential key regulators of human diseases, including cancer $(2,3)$. The functions and mechanisms of action of long ncRNA (lncRNA), transcripts ranging from 200 nucleotides to $100 \mathrm{~kb}$, are also being uncovered, in applications ranging from cancer to epigenetics (4-8). Some of these lncRNA are expressed in a cellular compartment- or tissue-specific manner and at substantially lower levels than mRNAs (9), which suggests key regulatory roles in gene expression both at transcriptional and post-transcriptional levels (10-12).

Malat1, also known as non-coding nuclear-enriched abundant transcript 2 (Neat2), was originally identified as an IncRNA whose expression was increased in early-stage non-small cell lung cancers that later metastasized compared with those that did not progress into tumors (13). Malat1 lacks open reading frames of significant length, is highly conserved and is located in the nucleus, specifically in nuclear speckles (14), where it regulates alternative splicing (15). In addition, post-transcriptional modifications of Malat1 yield a tRNA-like $61 \mathrm{nt}$ transcript (termed mascRNA) that is exported to the cytoplasm (16) for as yet elusive functions.

High expression levels of Malat1 within tumors have been associated with poor prognosis and severity of various types of cancer, including bladder (17), lung (18), gallbladder (19) and liver cancers (20). In addition, circulating Malat1 levels have also been recently shown to predict development of hepatocellular carcinoma $(21,22)$, suggesting its possible use as a reliable biomarker. Moreover, Malat1 may be directly implicated in the development of cancer through its effect on cellular proliferation and its facilitating role in cell invasion and metastasis, two important hallmarks of cancer $(18,23,24)$. In cultured cells, Malat1 promotes proliferation and cell motility by influencing the expression of oncogenic transcription factors (e.g., B-MYB) or motility-related genes through transcriptional and/or post-transcriptional regulation $(23,25,26)$. Nevertheless, the direct causal contribution of Malat1 to carcinogenesis in vivo remains poorly investigated. In this study, we tested the hypothesis that absence of Malat1 impairs the development of liver carcinogenesis induced by the genotoxic agent DEN. Our results rather indicate that Malat1 ablation does not modify the susceptibility to DEN-associated hetapocarcinoma. 
A

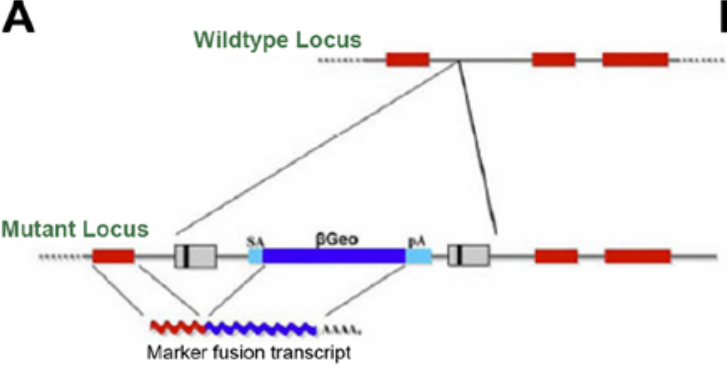

B

CAGGCATTCAGGCAGCGAGAGCAGAGCAGCGTAGAGCAGCACA GCTGAGCTCGTGAGGCAGGAGACTCAGCCCGAGGAAATCGCAG ATAAGTTTTTAATTAAAAAGATTGAGCAGTAAAAAGAATTAGA ACTCTAAACTTAAGCTAATAGAGTAGCTTATCGAAATATTACT TAGTCTTAATAATCTAAGAAGATCTTAAGAGATAACATGAAGG CTTATTTAAACAGTTTGAAAAAGGAAATGAGGAGA*AAAGTAT TTGTACTGTATAATGGAGGCTGACCAGAGCAGTTTAGGAGATT GTAAAGGGAGGTTTTGTGAAGTTCTAAAAGGTTCTAGTTTGAA GGTCGGCCTTGTAGATTAAAACGAAGGTTACCTAAATAGAATC TAAGTGGCATTTAAAACAGTAAAGTTGTAGAGAATAGTTTGAA AATGAGGTGTAGTTTTAAAAGATTGAGAAAAGTAGGTTAAGTT GACGGCCGTTATAAAAATCCTTCGACTG

C

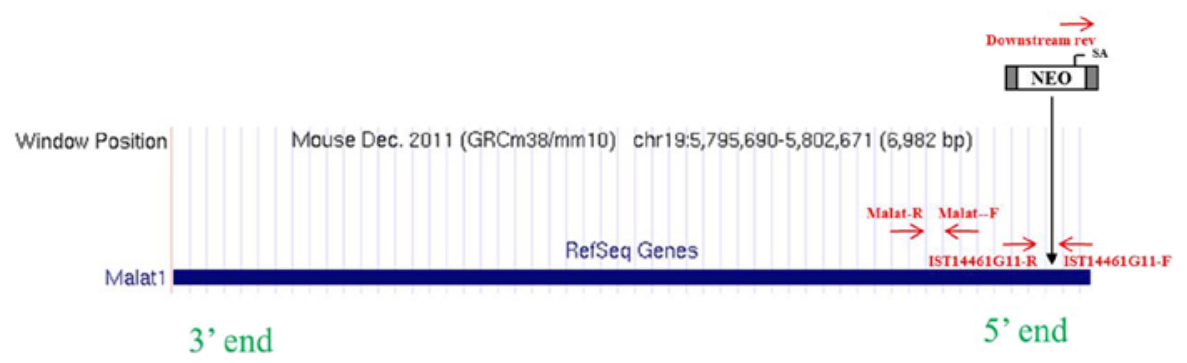

D
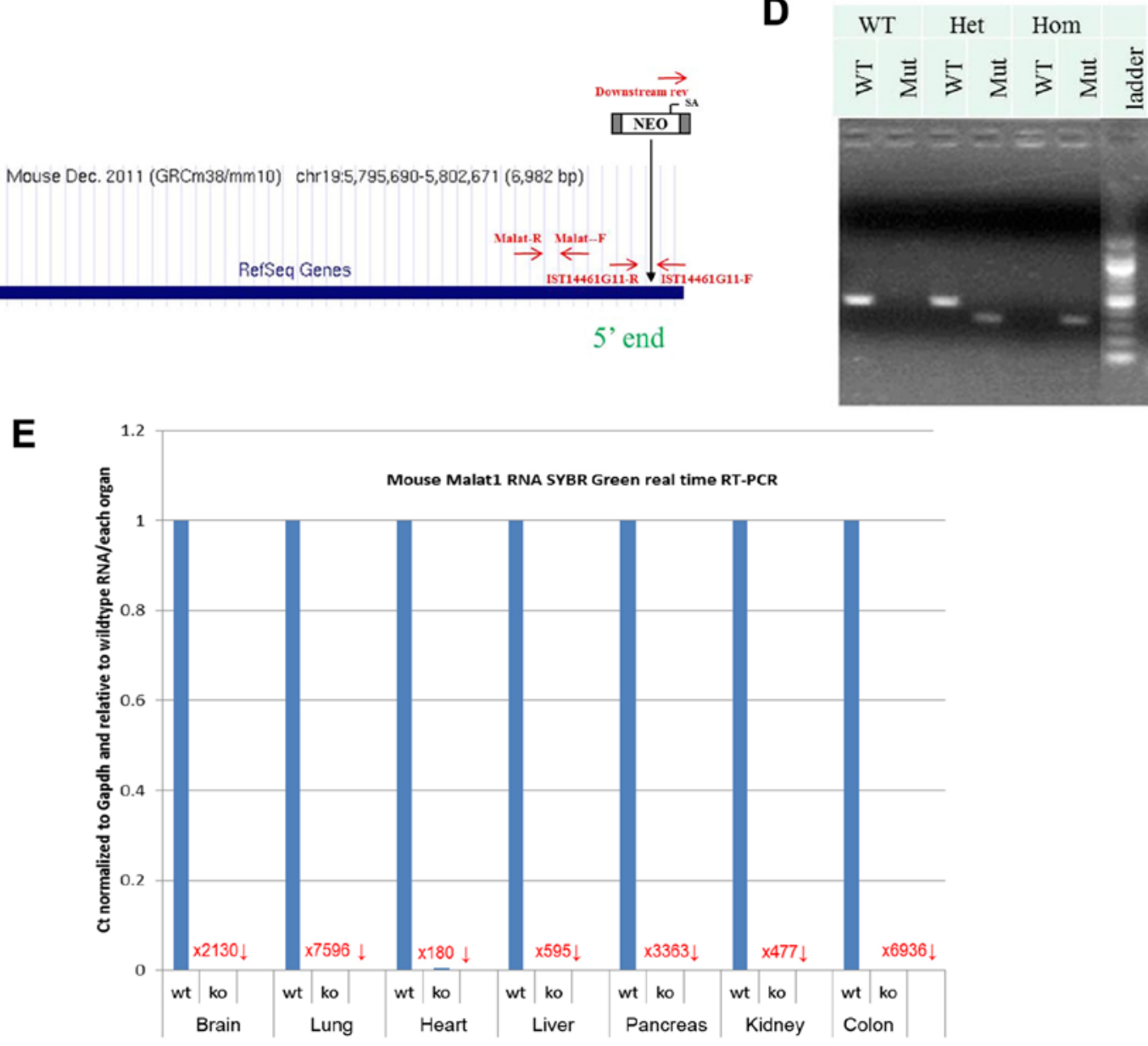

Figure 1. Generation of the Malat1 knockout mice used in this study. (A) Basic strategy of the gene trap vectors used in C57BL/6 library ( $\beta$-geo version shown). LTR, long terminal repeat; SA, splice acceptor sequence; $\beta$-geo, galactosidase/neomycin phosphotransferase fusion gene; pA, polyadenylation sequence. (B) The TIGM ES cell clone IST14461G11, found to carry mutation in Malat1, was expanded and the genomic sequence surrounding the gene trap insertion site determined [the insertion site is denoted with an asterisk (*)]. (C) Schematic representation of the mutated Malat1 locus. IST14461G11-F, IST14461G11-R and downstream rev are the primers used in genotyping [(C) and Table II]. Malat1-F and Malat1-R are primers used to confirm knockout via RT-PCR [(E) and Table II]. (D) Genotyping results showing wild-type (WT), heterozygous (Het) and homozygous (Hom) mutants using primer sets for wild-type (WT) and mutant (Mut) alleles in TIGM clone IST14461G11 for Malat1. (E) Fold change in Malat1 gene expression in knockout mice as compared to WT littermates. Assay performed with $20 \mathrm{ng}$ total RNA. The assay was performed using a primer pair amplifying a 170-nt fragment of the transcript downstream of the gene trap insertion (C). Expression levels were normalized to GAPDH expression.

\section{Materials and methods}

Animals. Mutant Malat1 mice were generated using a genetrapping technique (27) (Fig. 1A). Mice (strain C57BL/6) were cloned from an ES cell line (IST14461G11; Texas A\&M Institute for Genomic Medicine, TIGM). The ES cell clone contained a retroviral insertion in the Malatl gene identified from the TIGM gene trap database (Fig. 1B), and was microinjected into C57BL/6 host blastocysts to generate germline chimeras using standard procedures (28). The retroviral OmniBank Vector 74 contained a splice acceptor sequence (SA) followed by a 5 ' selectable marker neomycin resistance 
Table I. Primers used for the quantification of genes detailed in Fig. 5.

\begin{tabular}{lll}
\hline Gene name & \multicolumn{1}{c}{ Forward } & \multicolumn{1}{c}{ Reverse } \\
\hline HPRT & AAACTTTGCTTTCCCTG & AGGCTTTGTATTTGGCT \\
Ki67 & AGGAGGCAGCTAAGGACACA & ACACTTCCTTGGGGTCCTCT \\
P53 & AGAGACCGCCGTACAGAAGAAG & CAGCGTCGGCCTCGTCAGTC \\
HDAC3 & CACCCGCATCGAGAATCAGAAC & GATGCTCTGCTCGATGACAA \\
TERT & AGGGTAAGCTGGTGGAGGTT & CCAGCCCCAATGTCCCGTAGG \\
HDAC1 & ACGGGAGGCTCTGTCGCAAGTG & AGTCCCCGCGCTGCTCCTCTAT \\
NFkB & GCTCAGCGGGCAGTATTCCT & CACGTTCCGGCGGGCATTCTGG \\
Foxo3 & GGCTCCCCAACCGGCTCCTTCAA & GTGGTCGAGTTGGACTGGTT \\
FoxoA1 & CTCCCGGTACTTCTCTGCTG & GCGCGGGGGCCTGTAGTAGAAC \\
P27 & CGAGCCTGGAGCGGATGGAC & ATCCCAGATAAGCCCACCCC \\
P21 & CAGGTCGGCAGGAGGCATATCTAG & CGGACTCCGCAAAGTCTAAG \\
TNF $\alpha$ & AACTAGTGGTGCCAGCCGATG & AGGGGCATCGTCGTGGCTGTCTG \\
CDK4 4 & CTCGCCGCCGCTGGGAAACTT & CACGGGTGTTGCGTATGTAG \\
IL-6 & GTCAGTTTCTAAGCGGCCTG & CAGAATTGCCATTGCACAAC \\
\hline
\end{tabular}

genes, for identification of successful gene trap events followed by a polyadenylation signal (pA) (Fig. 1C). Insertion of the retroviral vector into the Malatl gene led to the splicing of the endogenous upstream exons into this cassette to produce a fusion transcript and terminate expression of the RNA downstream. Chimeric males were bred to C57BL/6 females for germline transmission of the mutant Malatl allele. The correct mutation was confirmed using PCR-based genotyping protocol using primers specific for genomic insertion site and for the vector 5'-AGAGCAGAGCAGCGTAGAGC-3', 5'-TAACG GCCGTCAACTTAACC-3', 5'-CCAATAAACCCTCTTGCA GTTGC-3' (Fig. 1D). Malat1 expression levels were quantified in several tissues to confirm gene knockout (Fig. 1E). Compared to wild-type littermates, Malat $1^{-/-}$mice showed an absence of Malat1 expression in all tissues studied (Fig. 1E). Notably, expression in the liver was reduced by 595 -fold (Fig. 1E).

At 15-day-old, wild-type (WT) and Malat ${ }^{-/-}$male littermates were injected with $5 \mu \mathrm{g} / \mathrm{g}$ of the potent genotoxic agent N-nitrosodiethylamine (DEN) (Sigma no. N0258) to induce liver tumors (29). Mice were weighed every day for the first 15 days and then weekly for the remaining of the protocol. Mice were exposed to a $12: 12$-h dark-light cycle and kept at ambient temperature of $\pm 2{ }^{\circ} \mathrm{C}$. Animals were euthanized by exsanguination (cardiac puncture) under ketamine/xylazine anesthesia. Tissues were collected, weighed, and snap frozen or fixed in 4\% paraformaldehyde (PFA) until further processing. All mice were cared for and handled in conformance with the Canadian Guide for the Care and Use of Laboratory Animals, and protocols were approved by our institutional animal care committee.

Histology. Scoring of histologic parameters was performed by an anatomic pathologist with experience in pulmonary pathology, independently and blinded to experimental data, using an Olympus BX53 microscope. A semiquantitative scale was used to score bronchial/endobronchial, peribronchial, perivascular, interstitial, pleural and intra alveolar inflammation, capillary vascular congestion and pulmonary edema. When present, metastases were measured and photographed.

Plasma biochemistry. At sacrifice, blood was collected by intracardiac puncture and placed into a tube containing EDTA. Plasma was stored at $-80^{\circ} \mathrm{C}$ for further biochemical analyses. Plasma cholesterol and triglycerides were measured using colorimetric assays (Thermo Fisher Scientific and Wako, respectively).

Glucose tolerance test. To evaluate glucose tolerance, mice were fasted for $12 \mathrm{~h}$ starting at $8 \mathrm{pm}$ with free access to water. The following morning, mice were weighed, baseline glycemia was measured and mice were injected intraperitoneally with $2 \mathrm{~g} / \mathrm{kg}$ of D-glucose. Glycemia was measured in blood from the tail vein at different intervals following glucose injection using an Accu-Chek performa glucometer (Roche).

RNA extraction and real-time quantitative PCR analysis. Total RNA was extracted using Aurum total RNA fatty and fibrous tissue kit (Bio-Rad). Purity, degradation state and concentration of the RNA samples were analyzed by the Experion automated electrophoresis system (Bio-Rad). cDNA was synthetized from $1 \mu \mathrm{g}$ of RNA using qScript reverse transcriptase (Quanta Bioscience, USA) according to the manufacturer's instructions. Semi-quantitative PCR was carried using an ABI 7900. Chemical detection of the PCR products was achieved with SYBR Green Jumpstart Taq ReadyMix without $\mathrm{MgCl}_{2}$ (Sigma, Oakville, ON, USA) (30). All reactions were performed in duplicate and relative level of gene expression was determined by the standard curve method. Results were normalized to the expression level of the reference gene hypoxanthine-guanine phosphoribosyltransferase (HPRT), which did not differ between groups. PCR primers used are listed in Tables I and II. 
Table II. Primer used to assess the reduction in Malat1 expression in Malat1 knockout animals (Fig. 1E).

\begin{tabular}{lll}
\hline Gene name & \multicolumn{1}{c}{ Forward } & \multicolumn{1}{c}{ Reverse } \\
\hline Malat1 & $\begin{array}{l}\text { GGCAGAATGCCTTTGAAGAG } \\
\text { (named Malat1-F in Fig. 1C) }\end{array}$ & $\begin{array}{l}\text { GGTCAGCTGCCAATGCTAGT } \\
\text { (named Malat1-R in Fig. 1C) }\end{array}$ \\
GAPDH & GGCATTGCTCTCAATGACAAC & GCCATGTAGGCCATGAGGT \\
\hline
\end{tabular}

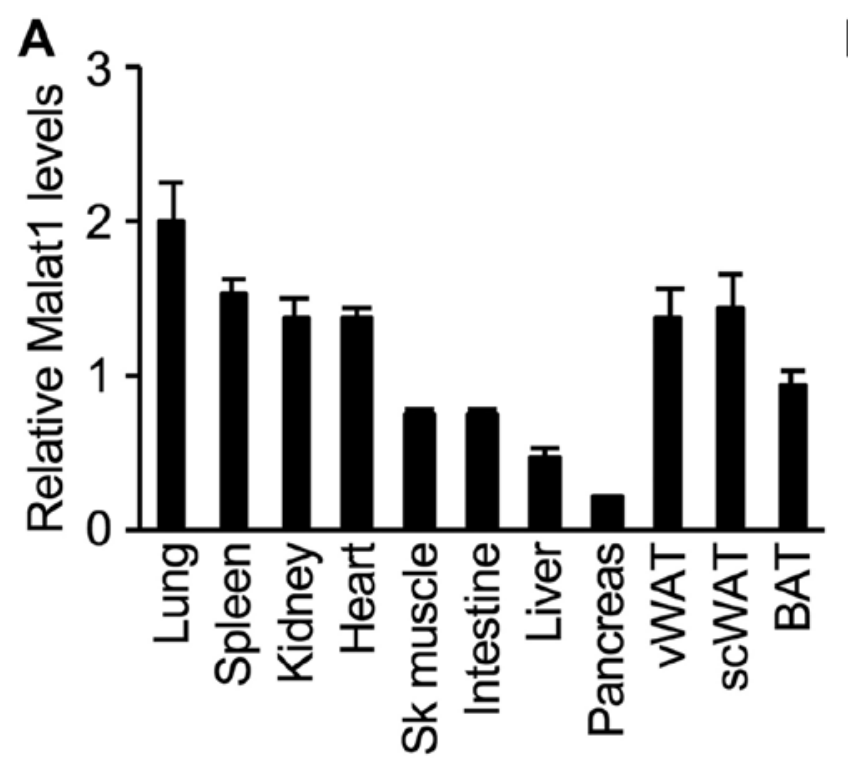

B

Figure 2. (A) Tissue expression of Malat1 in 8-week-old C57BL/6J male mice. Data normalized to $18 \mathrm{~S}$ levels. $\mathrm{n}=4$. Sk, skeletal; vWAT, epididymal white adipose tissue; scWAT, inguinal white adipose tissue; BAT, interscapular brown adipose tissue. (B) Malat1 expression levels in the liver of 14 months old WT mice one year after injection with saline or DEN. Measures performed on RNA extracted from tumors or healthy liver tissue. "Significant effect of tissue type (2-way ANOVA, $\mathrm{p}<0.05)$. n=3-5.

Data analysis. Data are presented as mean \pm SEM. Statistical differences were analyzed by Chi-square, ANOVA, ANOVA repeated measures, and Fisher's tests (ad hoc) when appropriate. A p-value $<0.05$ was considered statistically significant.

\section{Results}

In untreated 2-month-old WT male mice, hepatic expression levels of Malatl were lower when compared to those in other tissues (Fig. 2A). One year after DEN injection, an increase in Malat1 expression was observed in tumors when compared to that in healthy liver tissue (Fig. 2B). However, this increase in Malat1 levels was similar whether tumors developed spontaneously as function of age, or induced by DEN administration (Fig. 2B).

In WT mice, DEN injection did not change total liver weight (Fig. 3A), but resulted in an increased number and prevalence of tumors (Fig. 3B). This resulted in a distorted tissue with increased number of lobes containing at least one tumor (Fig. 3C). These features equally developed in DEN-treated Malat1 $^{-/}$animals (Fig. 3A-C) (no statistical difference between DEN-treated WT and Malat1 ${ }^{-/-}$mice), indicating that absence of Malat1 had no impact on the incidence and severity of DEN-induced hepatocarcinoma.
Histopathology examination of the lung and semi-quantitative histologic scaling between DEN-treated WT mice and Malat $^{-/-}$littermates revealed comparable prevalence of peribronchial ( 2 mice vs 0 , respectively), perivascular (2 vs 4) interstitial ( 0 vs 0 ), pleural (0 vs 0 ), and intra-alveolar inflammation (1 vs 0 ). One DEN-treated WT mouse had atypical cell foci suggesting a lung metastasis, whereas one Malat ${ }^{-/-}$mouse had well-defined, differentiated lung metastasis (Fig. 3D).

Beyond structural damage, deletion of Malat1 may have modulated hepatic metabolic functions upon DEN administration. Although DEN had no impact on plasma cholesterol levels in WT mice, it unexpectedly increased 2-fold those of Malat1 $^{-/}$littermates (Fig. 4A). In contrast, no difference in triglyceride levels (Fig. 4B) or glucose tolerance (Fig. 4C) was observed between groups.

Finally, the expression of genes involved in cell cycle and inflammation was quantified in liver tumors. Compared to their WT littermates, Malat $1^{-/-}$mice showed significantly lower hepatic mRNA levels of Ki67, TERT, HDAC1, NFкB, Foxo3, p27, and IL-6 (Fig. 5). DEN treatment diminished TERT mRNA levels but increased those of IL-6 (Fig. 5). No significant interaction between genotype and DEN administration was observed, indicating that absence of Malatl did not modify the transcriptional response to DEN in hepatic tumors. 
A

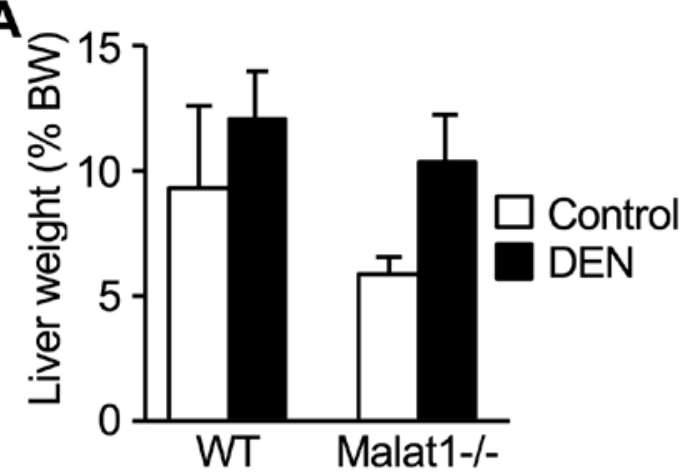

C

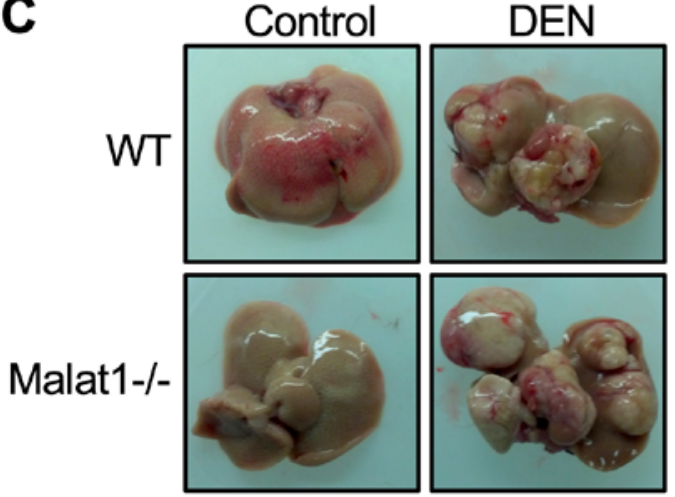

B

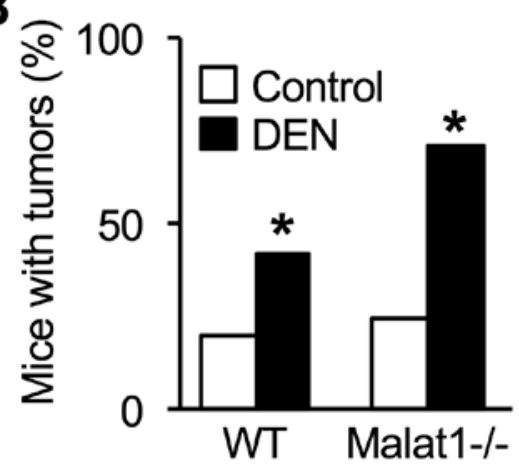

D
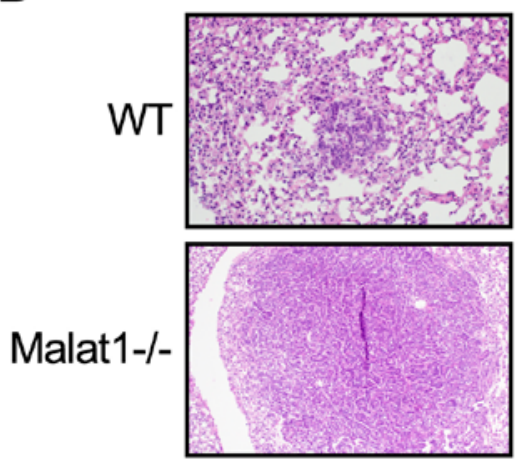

Figure 3. (A) Liver weight in WT and Malat ${ }^{-/}$male mice one year after saline or DEN injections. $n=6$. (B) Percentage of mice described in (A) with at least one tumor. "Significant effect of DEN compared to control saline group (Chi-square test, $p<0.05$ ). $n=6$. (C) Representative images of liver from mice described in (A). (D) Upper, from a DEN-treated WT mouse: microscopic foci of atypical cells with intra-alveolar macrophages and abundant cytoplasm, suspicious for a lung metastasis from a well-differentiated hepatocarcinoma. Size of the lesion, $0.2 \mathrm{~mm}$ (x200). Lower, from a DEN-treated Malat $1^{-/}$mouse: lung metastasis from a well-differentiated hepatocarcinoma. Size of the lesion, $1.5 \mathrm{~mm}$ (x100).

A

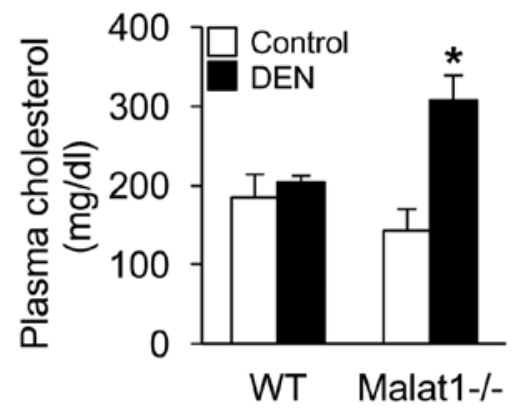

C

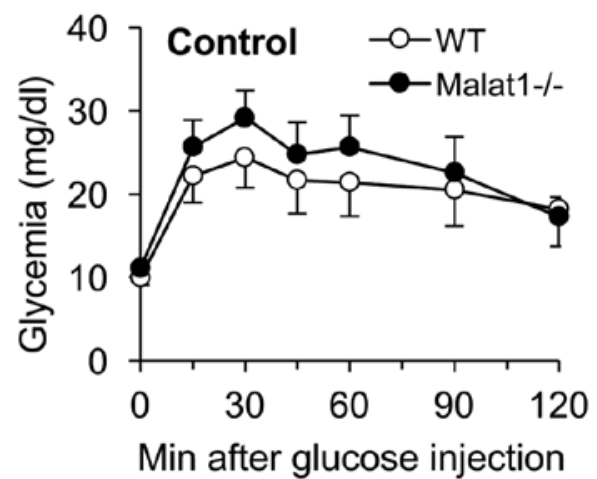

B

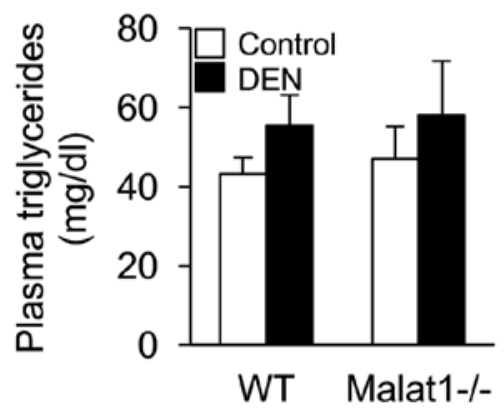

D

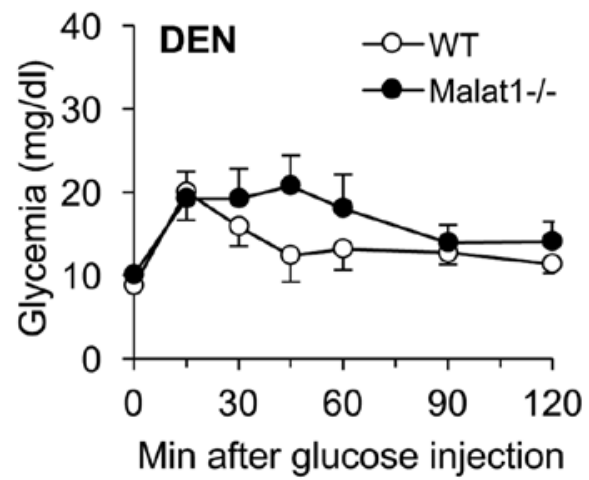

Figure 4. (A) Plasma cholesterol and (B) triglycerides levels in overnight fasted mice one year after saline or DEN injections. Data are presented as mean \pm SEM. *Significant effect of DEN compared to respective saline group (Fisher's post hoc test, $\mathrm{p}<0.05$ ). $\mathrm{n}=4$. (C) Glucose tolerance test in saline-treated mice. $n=3-5$. (D) Glucose tolerance test in DEN-treated mice. $n=3-5$. 


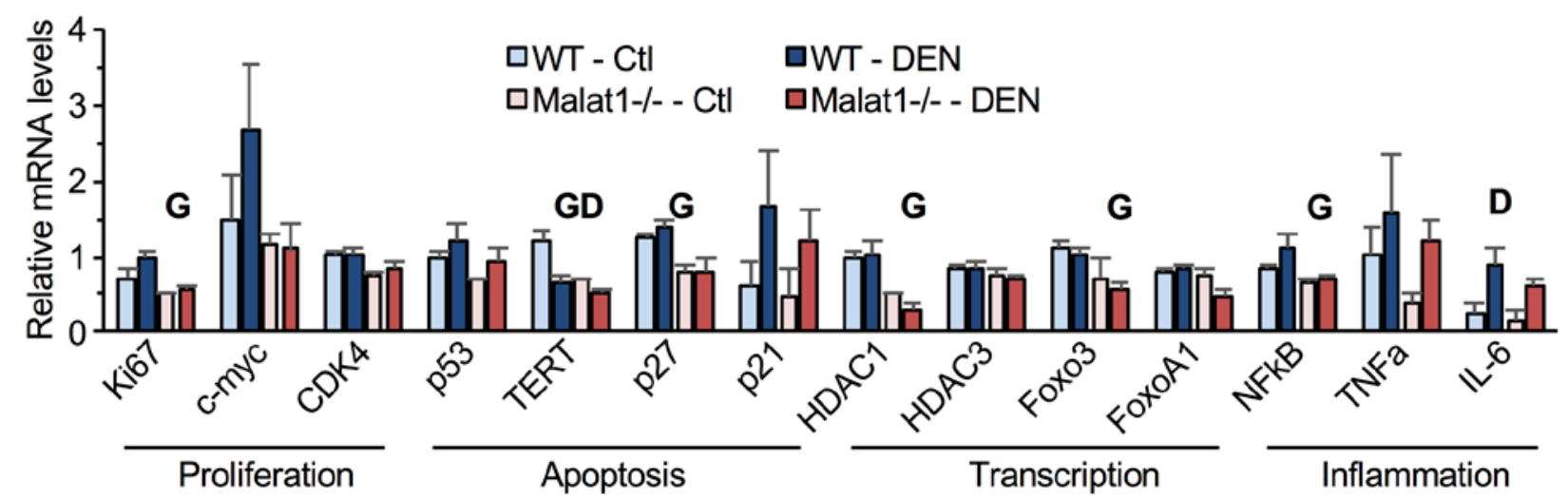

Figure 5. Gene mRNA levels in liver of WT and Malat $1^{-/}$mice one year after saline or DEN injections. Measures performed on RNA extracted from tumors. Bars represent mean \pm SEM. G and D indicate a statistically significant difference $(\mathrm{p}<0.05)$ main effect of genotype and DEN, respectively, as analyzed by 2-way ANOVA. No G x D interaction detected. $n=3-6$.

\section{Discussion}

Many studies performed in cultured cells have demonstrated a stimulating role of Malat1 on cancer cell proliferation $(20,31,32)$. Malat1 expression is increased during normal cell cycle progression in normal diploid human fibroblasts and has been found important for G1/S and mitotic division (23). Accordingly, Malat1 is overexpressed in various cancer types including lung, liver and breast cancer, and is associated with a poor prognostic (26). Moreover, Malat1 overexpression has been shown to predict tumor recurrence of hepatocellular carcinoma after liver transplantation (20). Despite these findings, the role of Malat1 in tumorigenesis in vivo is still not established. This study tested whether genetic deletion of Malat1 in mice impaired the development of hepatocarcinoma induced by the genotoxic agent DEN, a well-known regimen for the induction of hepatocellular carcinoma (29).

Compared to its levels in many tissues, Malat1 expression was low in liver of young and healthy WT mice. In tumors, Malat1 was 2- to 3-fold higher than in healthy liver tissue; however, no further increase was observed upon DEN treatment. This indicates that Malat1 expression may have reached a plateau in actively proliferating hepatocytes, perhaps due to mutual inhibition by YAP and SRSF1 (33). In this context, it was thus expected that genetic ablation of Malat1 would in theory translate into large, physiologically relevant impacts in a model in which increased expression of Malat1 normally occurs. However, the main finding of this study is that Malat $1^{-1-}$ mice are as susceptible to DEN-induced liver cancer as WT mice. Beyond this, Malat1 may be implicated in the development of cancer through its involvement in facilitating invasion and metastasis $(23,24,34)$. However, we found no significant difference in the prevalence of pulmonary inflammation or lung metastases between WT and Malat $1^{-1-}$ mice. This could be attributable to the experimental regimen, as DEN is a powerful genotoxic agent that may bypass some of the tumorigenic pathways triggered by Malat1. In this view, it would be interesting to test the influence of Malat1 in other tumor-inducing contexts, such as tobacco-induced lung cancer.

Genetic deletion of Malat1 (complete germline knockout) in mice has very little impact on pre- and post-natal devel- opment and on adult phenotypes when tested in normal conditions, as shown by three independent groups (35-38). The findings observed in the mouse line used herein also show that the basal modulation of glucose and lipid metabolism, functions regulated by the liver, were not affected by the absence of Malat1, at least in the fasted state. Although triglyceridemia and glucose tolerance were similar between WT and Malat $^{-/-}$littermates in response to DEN, plasma cholesterol levels were robustly increased in DEN-treated Malat $1^{-/-}$mice. Thus, Malat1 may play important roles in the regulation of cholesterol homeostasis. Interestingly, a recent report showed that, in contrast, Malat 1 expression is high in the fatty liver of obese ob/ob mice, and that it promotes cholesterol accumulation in HepG2 hepatocytes by increasing SREBP1-c protein stability (39). Thus, these observations suggest that Malat1 may exert beneficial impacts on cholesterol metabolism in a manner dependent upon conditions in which liver functions are perturbed. The mechanisms for these divergent effects could be independent from obesity-associated, IL-6-induced liver inflammation and tumorigenesis (40), since Malat1 deficiency did not modify the increase in IL-6 expression triggered by DEN treatment. Therefore, the role of Malat1 on cholesterol homeostasis remains to be established in vivo in different settings, including non-alcoholic fatty liver disease (NAFLD).

In vitro $(17,25,32)$ and in a mouse model of xenografts (18), Malat1 was shown to stimulate cytoskeleton components, cell proliferation, and cellular motility of cancer cells through impacting gene transcription, not alternative splicing per se (18). Consistent with these findings, our study shows that absence of Malat1 results in a significant downregulation of many genes involved in cell cycle and inflammation. Nonetheless, the observed changes in gene expression were not sufficient to induce a robust and specific phenotype. It remains to be investigated whether knockout of Malatl modified the expression of genes through cis-regulatory mechanism as previously reported in other cell types (38).

A study performed in metastatic renal cell carcinoma has suggested Malat1 as a putative FoxP3 target gene (41). Interestingly, this family of transcription factors has been shown to be involved in the sexual dimorphism observed in 
the development of liver cancer (42). Since female mice are not affected by DEN exposure (29), it would be interesting to test whether this dimorphism exists in Malat $1^{-/}$mice using other types of carcinogens or by crossing Malat $1^{-/-}$mice with susceptible transgenic strains.

In conclusion, we hypothesized that absence of Malat1 would confer resistance to liver carcinogenesis induced by DEN. As expected, DEN treatment stimulated an increase in liver tumors, and number of lobes with at least one tumor. However, these changes were completely similar to those observed in Malat1 ${ }^{-/}$mice, despite differences in their transcriptional mRNA profiles. In conclusion, gene deletion of Malatl does not impact cell proliferation upon DEN-induced hepatocarcinoma in vivo. Thus, in this model, the role of Malat1 in the regulation of hepatocyte proliferation is either minimal or masked by redundant and/or overwhelming mechanisms, not present in in vitro settings, including hormonal cues. Since Malat1 has been found to be highly upregulated in many other types of cancer, the impact of Malatl deficiency to the in vivo development of these diseases remains to be investigated.

\section{Acknowledgements}

This study was supported by a grant from the Natural Sciences and Engineering Research Council (NSERC) of Canada to F.P. M.J.G. was a recipient of an MSc studenship from the Fonds d'enseignement et de recherche - FER from Laval University/ Faculty of Pharmacy. S.C. is the recipient of a PhD studentship award from the Fonds de Recherche du Québec-Santé (FRQS). F.P. holds a Senior Scholar Award from the FRQS.

\section{References}

1. Ankö ML and Neugebauer KM: Long non-coding RNAs add another layer to pre-mRNA splicing regulation. Mol Cell 39: 833-834, 2010

2. Rottiers V and Näär AM: MicroRNAs in metabolism and metabolic disorders. Nat Rev Mol Cell Biol 13: 239-250, 2012.

3. Stefani G and Slack FJ: Small non-coding RNAs in animal development. Nat Rev Mol Cell Biol 9: 219-230, 2008.

4. Li J, Meng H, Bai Y and Wang K: Regulation of lncRNA and its role in cancer metastasis. Oncol Res 23: 205-217, 2016.

5. Dhamija S and Diederichs S: From junk to master regulators of invasion: lncRNA functions in migration, EMT and metastasis. Int J Cancer 139: 269-280, 2016.

6. Betancur JG: Pervasive lncRNA binding by epigenetic modifying complexes - The challenges ahead. Biochim Biophys Acta 1859: 93-101, 2016.

7. Blythe AJ, Fox AH and Bond CS: The ins and outs of lncRNA structure: How, why and what comes next? Biochim Biophys Acta 1859: 46-58, 2016.

8. Sun M, Nie FQ, Wang ZX and De W: Involvement of lncRNA dysregulation in gastric cancer. Histol Histopathol 31: 33-39, 2016.

9. Kaikkonen MU, Lam MT and Glass CK: Non-coding RNAs as regulators of gene expression and epigenetics. Cardiovasc Res 90: 430-440, 2011.

10. Rinn JL and Chang HY: Genome regulation by long non-coding RNAs. Annu Rev Biochem 81: 145-166, 2012.

11. Ponting CP, Oliver PL and Reik W: Evolution and functions of long non-coding RNAs. Cell 136: 629-641, 2009.

12. Wilusz JE, Sunwoo H and Spector DL: Long non-coding RNAs: Functional surprises from the RNA world. Genes Dev 23: 1494-1504, 2009.

13. Ji P, Diederichs S, Wang W, Böing S, Metzger R, Schneider PM, Tidow N, Brandt B, Buerger H, Bulk E, et al: MALAT-1, a novel non-coding RNA, and thymosin beta4 predict metastasis and survival in early-stage non-small cell lung cancer. Oncogene 22: 8031-8041, 2003.
14. Hutchinson JN, Ensminger AW, Clemson CM, Lynch CR, Lawrence JB and Chess A: A screen for nuclear transcripts identifies two linked non-coding RNAs associated with SC35 splicing domains. BMC Genomics 8: 39, 2007.

15. Tripathi V, Ellis JD, Shen Z, Song DY, Pan Q, Watt AT, Freier SM, Bennett CF, Sharma A, Bubulya PA, et al: The nuclear-retained non-coding RNA MALAT1 regulates alternative splicing by modulating SR splicing factor phosphorylation. Mol Cell 39: 925-938, 2010.

16. Wilusz JE, Freier SM and Spector DL: $3^{\prime}$ end processing of a long nuclear-retained non-coding RNA yields a tRNA-like cytoplasmic RNA. Cell 135: 919-932, 2008.

17. Ying L, Chen Q, Wang Y, Zhou Z, Huang Y and Qiu F: Upregulated MALAT-1 contributes to bladder cancer cell migration by inducing epithelial-to-mesenchymal transition. Mol Biosyst 8: 2289-2294, 2012.

18. Gutschner T, Hämmerle M, Eissmann M, Hsu J, Kim Y, Hung G, Revenko A, Arun G, Stentrup M, Gross M, et al: The non-coding RNA MALAT1 is a critical regulator of the metastasis phenotype of lung cancer cells. Cancer Res 73: 1180-1189, 2013.

19. Wang SH, Zhang WJ, Wu XC, Zhang MD, Weng MZ, Zhou D, Wang JD and Quan ZW: Long non-coding RNA Malatl promotes gallbladder cancer development by acting as a molecular sponge to regulate miR-206. Oncotarget 7: 37857-37867, 2016.

20. Lai MC, Yang Z, Zhou L, Zhu QQ, Xie HY, Zhang F, Wu LM, Chen LM and Zheng SS: Long non-coding RNA MALAT-1 overexpression predicts tumor recurrence of hepatocellular carcinoma after liver transplantation. Med Oncol 29: 1810-1816, 2012.

21. Luo F, Sun B, Li H, Xu Y, Liu Y, Liu X, Lu L, Li J, Wang Q, Wei S, et al: A MALAT1/HIF-2 $\alpha$ feedback loop contributes to arsenite carcinogenesis. Oncotarget 7: 5769-5787, 2016.

22. Konishi H, Ichikawa D, Yamamoto Y, Arita T, Shoda K, Hiramoto H, Hamada J, Itoh H, Fujita Y, Komatsu S, et al: Plasma level of metastasis-associated lung adenocarcinoma transcript 1 is associated with liver damage and predicts development of hepatocellular carcinoma. Cancer Sci 107: 149-154, 2016.

23. Tripathi V, Shen Z, Chakraborty A, Giri S, Freier SM, Wu X, Zhang Y, Gorospe M, Prasanth SG, Lal A, et al: Long non-coding RNA MALAT1 controls cell cycle progression by regulating the expression of oncogenic transcription factor B-MYB. PLoS Genet 9: e1003368, 2013.

24. Hanahan D and Weinberg RA: Hallmarks of cancer: The next generation. Cell 144: 646-674, 2011.

25. Tano K, Mizuno R, Okada T, Rakwal R, Shibato J, Masuo Y, Ijiri K and Akimitsu N: MALAT-1 enhances cell motility of lung adenocarcinoma cells by influencing the expression of motilityrelated genes. FEBS Lett 584: 4575-4580, 2010.

26. Gutschner T, Hämmerle M and Diederichs S: MALAT1 - a paradigm for long non-coding RNA function in cancer. J Mol Med (Berl) 91: 791-801, 2013.

27. Hansen GM, Markesich DC, Burnett MB, Zhu Q, Dionne KM, Richter LJ, Finnell RH, Sands AT, Zambrowicz BP and Abuin A: Large-scale gene trapping in C57BL/6N mouse embryonic stem cells. Genome Res 18: 1670-1679, 2008.

28. Hogan B, Beddington R, Costantini, F and Lacy E: Manipulating the Mouse Embryo: A Laboratory Manual. Cold Spring Harbor Laboratory Press, New York, NY, 1994.

29. Fausto $N$ and Campbell JS: Mouse models of hepatocellular carcinoma. Semin Liver Dis 30: 87-98, 2010.

30. Miard S, Dombrowski L, Carter S, Boivin L and Picard F: Aging alters PPARgamma in rodent and human adipose tissue by modulating the balance in steroid receptor coactivator-1. Aging Cell 8: 449-459, 2009

31. Guo F, Li Y,Liu Y, Wang J, Li Y and Li G: Inhibition of metastasisassociated lung adenocarcinoma transcript 1 in CaSki human cervical cancer cells suppresses cell proliferation and invasion. Acta Biochim Biophys Sin (Shanghai) 42: 224-229, 2010.

32. Han Y, Liu Y, Nie L, Gui Y and Cai Z: Inducing cell proliferation inhibition, apoptosis, and motility reduction by silencing long non-coding ribonucleic acid metastasis-associated lung adenocarcinoma transcript 1 in urothelial carcinoma of the bladder. Urology 81: 209.e201-207, 2013.

33. Wang J, Wang H, Zhang Y, Zhen N, Zhang L, Qiao Y, Weng W, Liu X, Ma L, Xiao W, et al: Mutual inhibition between YAP and SRSF1 maintains long non-coding RNA, Malat1-induced tumourigenesis in liver cancer. Cell Signal 26: 1048-1059, 2014.

34. Gutschner T and Diederichs S: The hallmarks of cancer: A long non-coding RNA point of view. RNA Biol 9: 703-719, 2012 . 
35. Nakagawa S, Naganuma T, Shioi G and Hirose T: Paraspeckles are subpopulation-specific nuclear bodies that are not essential in mice. J Cell Biol 193: 31-39, 2011.

36. Nakagawa S, Ip JY, Shioi G, Tripathi V, Zong X, Hirose T and Prasanth KV: Malat1 is not an essential component of nuclear speckles in mice. RNA 18: 1487-1499, 2012.

37. Eissmann M, Gutschner T, Hämmerle M, Günther S, CaudronHerger M, Gross M, Schirmacher P, Rippe K, Braun T, Zörnig M, et al: Loss of the abundant nuclear non-coding RNA MALAT1 is compatible with life and development. RNA Biol 9: 1076-1087, 2012.

38. Zhang B, Arun G, Mao YS, Lazar Z, Hung G, Bhattacharjee G, Xiao X, Booth CJ, Wu J, Zhang C, et al: The lncRNA Malat1 is dispensable for mouse development but its transcription plays a cis-regulatory role in the adult. Cell Rep 2: 111-123, 2012.
39. Yan $\mathrm{C}$, Chen $\mathrm{J}$ and Chen $\mathrm{N}$ : Long non-coding RNA MALAT1 promotes hepatic steatosis and insulin resistance by increasing nuclear SREBP-1c protein stability. Sci Rep 6: 22640, 2016.

40. Park EJ,Lee JH, Yu GY,He G, Ali SR, Holzer RG,OsterreicherCH, Takahashi $\mathrm{H}$ and Karin M: Dietary and genetic obesity promote liver inflammation and tumorigenesis by enhancing IL-6 and TNF expression. Cell 140: 197-208, 2010.

41. Schwarzer A, Wolf B, Fisher JL, Schwaab T, Olek S, Baron U, Tomlinson CR, Seigne JD, Crosby NA, Gui J, et al: Regulatory T-cells and associated pathways in metastatic renal cell carcinoma (mRCC) patients undergoing DC-vaccination and cytokine-therapy. PLoS One 7: e46600, 2012.

42. Li Z, Tuteja G, Schug J and Kaestner KH: Foxa1 and Foxa2 are essential for sexual dimorphism in liver cancer. Cell 148: 72-83, 2012. 